\title{
Neuropsychological indicators of heteromodal cortex (dys)function relevant to conditioned blocking measures of attention in schizophrenia
}

\author{
Robert D. Oades ${ }^{1}$, Stefan Bender ${ }^{2}$, Bernhard W. Müller ${ }^{1,2}, \quad$ Gudrun Sartory $^{3}$ \\ 2001 Cognitive Neuropsychiatry, 6, 41-61.
}

1. Biopsychology Group, University Clinic for Child and Adolescent Psychiatry, Virchowstr. 174, 45147 Essen, Germany. 2. Biological Psychiatry Group, University Clinic for Adult Psychiatry, Virchowstr. 174, 45147 Essen, Germany. 3. University Department of Clinical Psychology, Max Horkheimerstr. 20, 42097 Wuppertal, Germany.

\begin{abstract}
Background. Learning a predictive relationship between two events can block learning about an added event (conditioned blocking, $\mathrm{CB}$ ). Patients with nonparanoid schizophrenia can show reduced $\mathrm{CB}$ and learn about the similar consequences of the added event. Methods. This study reports on the relationship of associative learning and $C B$ measures of attention to signs of heteromodal cortex function provided by performance on a battery of 10 neuropsychological tasks (i.e. frontal, parietal and temporal lobe function of the left and right hemisphere). Acquisition criteria for the task were achieved by 62 patients with schizophrenia and 62 matched controls but not by 39 other people with schizophrenia. Results. First right-hemisphere, visuo-spatial abilities were generally associated with faster task-learning, and patients that could not learn the task were poorer on tests emphasising setswitching abilities associated with left frontal lobe function. Second CB expression depended on Stroop- and Mooney-faces-task performance that is reported to require cingulate and parietal lobe function. Conclusions. As would be expected right hemisphere function was implicated in performing a visuospatial learning task. The additional $C B$ requirement incurred additional anterior cingulate and right parietal involvement. Functionally this probably reflected effortful attentional processes, and illustrates the problems of patients with schizophrenia in switching between automatic and controlled processing strategies.
\end{abstract}

Key words: Neuropsychology, Blocking, Schizophrenia, Attention, Prefrontal cortex, Parietal cortex

\section{Acknowledgements}

This research was supported by the German research council (DFG OA4/1-2). We are grateful to Dr. Jörg Wolstein, Luigi Fierro, Andrea Czwenk, Claudia Heeper, Isabelle Eisenbeis, and Angela Dolberg for assisting the authors in symptom rating and neuropsychological testing. We also thank Professor Christian Eggers and Professor Markus Gastpar for their interest and support.

\section{INTRODUCTION}

There are three major 'epicentres' in the brain for mediating and influencing the processes of selective attention. With special reference to spatial attention, yet independent of sensory modality, Mesulam argues that these centres include parts of frontal, cingulate and parietal cortices (Mesulam, 1999; Kim, Gitelman, Nobre, Parrish, LeBar, \& Mesulam, 1999). While the emphasis is on the frontal eye fields, posterior parietal and anterior cingulate cortices, he also explicitly points out that there are important contributions from the temp-oral lobes and several subcortical loci.

Disturbances of controlled attentional processes have long been known to be integral to schizophrenia (Callaway \& Naghdi, 1981) and more recently disruption of some automatic attentional processes have been reported for this illness (Straube and Oades, 1992). Studies of latent inhibition (LI) and conditioned blocking $(\mathrm{CB})$ have been carried out in an attempt to describe the disturbed 
selective attention-related function more closely (Gray, 1998; Oades, Zimmermann \& Eggers, 1996b, see below). But, to date these studies have not attempted to relate the specific disturbances of attention that they purport to measure with the activity of the underlying structures of the brain, as described by Mesulam and colleagues (above).

In this report we describe a first approximation of the cortical regions that may contribute to the expression of $\mathrm{CB}$. We report on the associations of $\mathrm{CB}$ performance with that recorded by the same subjects on a battery of neuro-psychological tests selected for their reflection of frontal, parietal and temp-oral lobe function in the left or right hemisphere. The rationale is that if a group of patients with a particular phenotype are impaired on both $C B$ and the performance of neuropsychological test $X$ (not $Y$ ) then one can propose the working hypothesis that the activity in the brain region associated with per-forming test $X$ may also contribute to the requirements for $C B$. However, first we introduce the $C B$ task, and then we elaborate the nature of the neuropsychologic tests and the brain regions with which the measures are putatively related.

\section{Conditioned Blocking (CB)}

CB, first described by Kamin (1969), is a measure of an aspect of the selective attention processes occurring during associative learning. It is recorded as the delay in learning that a stimulus (B) is associated with the same consequences as another stimulus (A) that is already becoming associated with these consequences. It appears that stimulus A "blocks" learning about the added yet redundant stimulus $B$. This delay is evident in comparison with a reference session where two stimuli are present from the start and the same subjects are able to learn about both. Latent inhibition (LI) refers to the delay in learning that a stimulus has consequences $(A+)$ when it has been experienced without consequences (A-). This delay (LI) is seen in comparison to the more rapid learning about the stimulus by other subjects when it is associated with consequences from the first exposure. Performance of both $\mathrm{CB}$ and $\mathrm{LI}$ have been interpreted in terms of enhanced selective processing of stimulus $\mathrm{A}$ or $\mathrm{A}+$ and the need to invoke controlled processes to learn about the change during learning. Thus studies of $C B$ are informative about normal strategies for the allocation of attention (e.g. to $A$ and not to $B$ ) and the circumstances for reduced $C B$ provide information about how redundant or irrelevant stimuli (e.g. B) can come to control response (see recent discussion in Holland, 1999). Reduced CB may explain the feeling of 'stimulus-overload' that patients with some types of schizophrenia describe.

The quest for the nature of the biological substrate associated with this process has been approached with the study of animals. With the use of brain lesions and treatment with mono-aminergic agents contributions of the nucleus accumbens and septohippocampal regions and of noradrenergic and dopaminergic systems to $C B$ have been described (Caza, 1984; Crider, Blockel, \& Solomon, 1986; Oades, Rivet, Taghzouti, Kharouby, Simon, \& Le Moal 1987; Rickert, Bennett, Lane, \& French, 1978). There is also evidence for a contribution to $C B$ expression of the mesocortical dopamine projection and the frontal cortex (Oades et a1., 1987). In contrast $\mathrm{LI}$ has been associated exclusively with mesolimbic structures and transmitters (review in Gray, 1998). In view of this and in the context of our examining (below) the neuropsychological associations of being able (or unable) to learn the task in which $\mathrm{CB}$ was presented, a further result from these animal studies is of interest. Treatment of the ventral tegmental area, the source of the meso-limbic and mesocortical paths, with a dopamine toxin resulted in the disruption of learning of the task on which $\mathrm{CB}$ was to be recorded (Oades et al., 1987).

CB develops in humans across puberty through adolescence to adulthood and, as with the animal studies, an association with catecholaminergic activity has been confirmed (Oades, Röpcke, \& Schepker, 1996a). The disruption of $C B$ in patients with a nonparanoid rather than paranoid schizophrenia has been reported (Oades et al., 1996b) and replicated (Oades, Müller, Bender, Sartory, 2000a). Further, normal CB was 
confirmed in healthy subjects treated with amphetamine, a model for paranoid schizophrenia (Gray, Pickering, Gray, Jones, Abrahams, \& Hemsley, 1997). In contrast, LI is reduced in patients with acute schizophrenia and is disrupted by amphetamine treatment of healthy subjects (see Gray, 1998; Kumari et al., 1999).

\section{Neuropsychology}

Tasks were selected for the neuropsychological battery for their implication of the function of structures located in the frontal, parietal or temporal lobes and centred more in the one or the other cerebral hemisphere. The intention for this study was to use a regression analysis to identify which neuropsychological performance(s) related to learning and to attentional abilities measured in the $C B$ task. If the performance of a specific neuropsychological task significantly contributes to an explanation of the variance of $a C B$ measure then it is a reasonable hypothesis that the brain region (s) implicated in the one, may also be implicated in the other function. It is therefore important to briefly review the reasons for attributing the neuropsychological tasks and functions selected to specific regions of the cerebral cortex.

Frontal lobe function is demonstrated by verbal fluency and trail making tests (TMT). Verbal fluency requires the production of as many words as possible beginning with a given letter (e.g. F, A or S, Benton \& Hamsher, 1989). Efficient performance depends on cognitive flexibility, retrieval and keeping track of production with short-term memory. Frontal brain damage, especially on the left, is specifically associated with deficits in production (Perret, 1974). Five studies using positron emission tomographic (PET) measures of cerebral blood flow (CBF) show activation centred on the left prefrontal and cingulate cortex, with de-activation in the superior temporal gyrus (Spence, Liddle, Stefan, Hellewell, Sharma et al. 2000 and references therein). Patients with schizophrenia produced fewer words, showed less activation and in areas that were more bilaterally distributed. In the TMT (form-B, Reitan, 1958) letters and numbers are distributed over a page and must be connected by lines drawn in an alternating and increasing sequence (e.g. A-1-B-2-C). The latency to completion in the form B-A corrects for basal perceptual and motor mechanisms in the simpler number-only task form $A$ and is a measure of the executive ability to switch between set (Hartje \& Poeck, 1997). Latencies to completion are markedly slowed after frontal brain-damage (Kolb \& Whishaw, 1996). Haemodynamic responses (fMRI) to switching in the sense of multiple simple discrimination task reversal were marked in several bilateral frontal areas (prefrontal, insular and supplementary motor cortex) as well as the cuneus and intraparietal sulcus (Dove, Pollmann Schubert, Wiggins \& von Cramon, 2000). TMT B-A scores are typically about $50 \%$ longer for patients with schizophrenia (Allen, Goldstein, \& Aldarondo, 1999). Poor TMT and verbal fluency performance is especially associated with the negative symptoms of schizophrenia (Liddle \& Morris, 1991; Verdoux, Liraud, Gonzales, Assens, Abalan et al., 1999)

The interference score on the Stroop test refers to the latency to name the colour in which a word is printed when the word denotes another colour. The interference latency is longer than that for naming colour patches or the meanings of word describing colour alone. It reflects the use of effort, inhibition and error correction (Botvinick, Nystrom, Fissell, Carter, \& Cohen, 1999) and is especially long in association with damage to the cingulate and left frontal cortex (Perret, 1974; Kolb \& Whishaw, 1996). These areas are activated for interference measures in healthy subjects (Cabeza \& Nyberg, 1997; Taylor, Kornblum, Lauber, Minoshima \& Koeppe, 1997). Unusually long interference latencies are not shown by many patients with schizophrenia, except those with symptoms of disorganisation, a symptom cluster that is particularly associated with $\mathrm{CBF}$ in the cingulate cortex (Liddle \& Morris, 1991).

The block-design test (Wechsler, 1981) consists of a square red and white pattern that is presented for replication from a number of its constituent parts. There are 9 trials of increasing difficulty. Longer than 
normal latencies are found after frontoparietal brain damage on the right (Warrington, James \& Maciejewski, 1986) and performance is associated with metabolic activation in this area (Chase, Fedio and Foster, 1984). Chase et al. (1984) also showed that parieto-temporal regions were activated by the picture completion task (Wechsler, 1981). In this task 17 pictures are presented with a feature missing (e.g., a door without a handle), which should be named by the subject within $20 \mathrm{sec}$. Abilities to switch between detailed and holistic aspects of selective perception are required.

In the modified Mooney Faces Closure Test (Mooney \& Ferguson, 1951; Lansdell, 1970) a subject categorises 47 degraded black-andwhite pictures of faces according to gender and age class (young, adult, old) presented in order of their difficulty and without a time limit. Accuracy measures Gestalt-like perceptual abilities (Lezak, 1995). Studies of the effects of brain damage implicate right parietal function. Brain activation studies have not been carried out with this task, but reports on local vs. global perceptual abilities and performance on ambiguous figures show bilateral activation in parieto-occipital regions (Fink, Halligan, Marshall, Frith, Frackowiak, \& Dolan, 1996; Kleinschmidt, Büchel, Zeki, \& Frackowiak, 1998).

Lastly, visual reproduction and logical memories were tested in the immediate and delayed form (Wechsler, 1987). In the former the subject draws geometric figures seen for $10 \mathrm{sec}$ immediately upon withdrawal of the items, or after $30 \mathrm{~min}$ : the latter task follows the same scheme but consists of a short story scored for accuracy of successive units of information in the text. The delayed forms are measures of visuo-spatial and verbal memory, respectively. Right temporal brain damage impairs delayed recall of non-verbal material (Delaney, Rosen, Mattson, Noelly, 1980) while left temporal brain damage impairs prose recall (Kolb \& Whishaw, 1996). Brain activation studies indicate that immediate episodic memory encoding and recall may have other correlates, involving the frontal lobe on the left for verbal material or bilaterally for non-verbal items (Hidaka, Sadato, Yamada, \& Yonekura, 2000).

In summary we examine the associations of neuropsychological performance and $C B$ in healthy subjects and patients with schizophrenia, where decreased $C B$ was associated with a nonparanoid diagnosis, low levels of thought disorder and prominent ideas-of-reference (Oades et al., 2000a). The specificity of these associations is controlled by a comparison with the neuropsychological performance of a sample of patients who did not acquire the associative learning task in which CB was presented. It would be expected that the visuo-spatial nature of the task presentation would be associated with tasks demonstrating right-hemispheric involvement. Left temporal lobe functions, as in logical memories, should not be implicated. In contrast, the selective attention requirement of $\mathrm{CB}$ should be associated with tasks demonstrating fronto-cingulate-parietal activation (see Mesulam, 1999, above), and not for example the delayed recall of visual patterns that reflects right temporal lobe functions.

\section{MethodS}

\section{Subjects}

For this study 101 patients meeting DSM-IV criteria for schizophrenia (American Psychiatric Association, 1994) were recruited from the child, adolescent and adult university psychiatry clinics and compared with 62 healthy controls recruited by advertisement and paid for their participation. The two groups were closely matched for age, education, socio-economic family status and hand-preference (Table 1).

All patients were admitted consecutively for functional psychosis and the initial diagnosis was made by the senior ward physician. They were re-examined for entry to the study by two senior physicians of the research group (DSM-IV, criteria A-E) and affective, schizoaffective and schizophreniform 
Table 1

\section{Demographic and Clinical Characteristics of the Subjects}

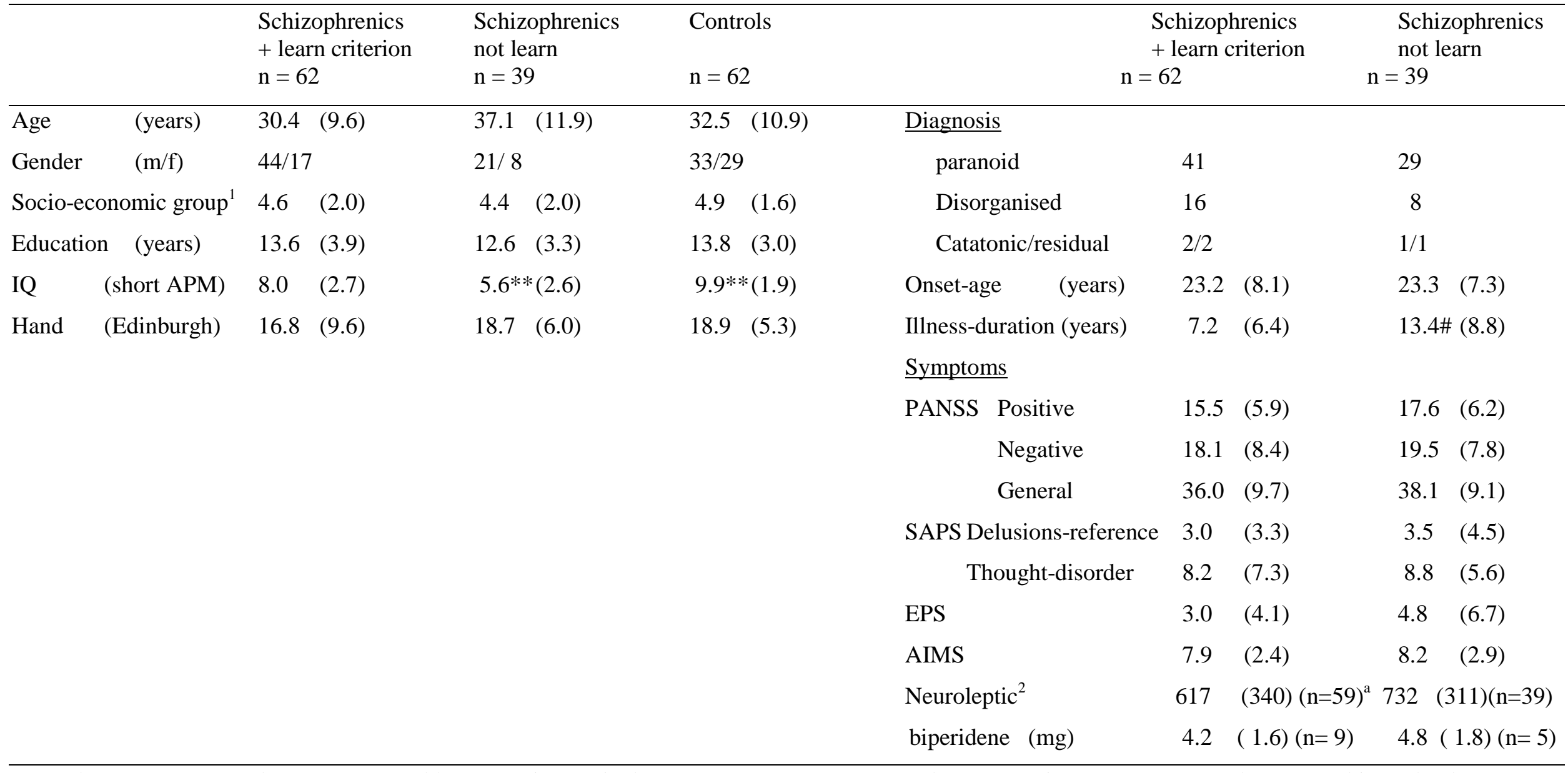

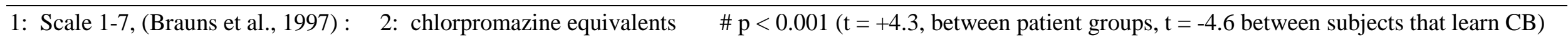
$* * \mathrm{p}<0.003$ (with respect to the patient group that learned) a: 2 medication-free, b: 32 vs. 15 on 'typical' \& 19 vs. 18 on clozapine or olanzapine, \& 9 vs. 6 on both typical and atypical neuroleptic 
psychoses were excluded. Patients were also screened to exclude other major psychiatric or somatic illness, alcohol abuse in the last 5 years and substance abuse other than nicotine. Schizophrenia subtypes were also defined by DSM-IV criteria, whereby the undifferentiated type was regarded as a residual category that contrasts with the paranoid, disorganised and catatonic subtypes (see Table 1 for clinical assessments and medication). The exclusion criteria for healthy participants, based on a semi-structured interview, were the same as described for the patients. In addition they reported no family history of psychotic illness, nor that they had previously consulted with a psychiatrist or psychologist.

Symptom severity was rated according to the Positive and Negative Syndrome Scale (PANSS: Kay, Opler \& Fizbein, 1992). Further, as Schneiderian ideas-of-reference (egodisturbance) and thought disorders are under represented in this rating scale, the relevant items from the Scale for Assessment of Positive Symptoms (SAPS: Andreasen \& Olsen, 1982) were also scored. Ratings of extrapyramidal symptoms were also included (Abnormal Involuntary Movement Scale, AIMS, (Simpson \& Angus, 1970) and the Barnes akathisia scale (1989). Handedness was evaluated with the Edinburgh inventory (Oldfield, 1971).

Illness-onset was defined as the age at onset of psychosis as assessed on interview with the patient along with information from a relative: for some patients this had to be set as the date of first admission (range 8.9$45.8 \mathrm{y}$ ). Illness-duration was taken as the time between illness-onset and testing (range 0.0220.1y). The current admission to the clinic had lasted from 1 to 211 days. At testing 2 patients were without medication, 40 received atypical (clozapine and olanzapine), 40 received more typical neuroleptics, and the rest were treated with both classes of medication (Table 1). Fourteen patients received biperidene (mean $3.9 \mathrm{mg}$, range 2-8 mg). Following approval from the medical centre ethics committee, informed signed consent was obtained from each patient and the responsible care-giver, and from each healthy participant.

\section{The Conditioned Blocking Task (CB)}

The $\mathrm{CB}$ task was presented as a computer game. A mouse-like cursor is moved with a joystick through a maze resembling the floorplan of an apartment (Fig. 1). Subjects started in pseudo-random order from the left or right sides of the maze and were asked to look in the other room for a goal (in mirror-image loci). On reaching the goal the locus would light up and 30 points were added to counters below the floor-plan. Every second over a latency of $8 \mathrm{sec}$ was scored with -1 point per second. These trials counted as 'errors' in the calculation of the learning criterion. The intertrial-interval was 2 seconds. The reaction time for skilled subjects was about $2.4 \mathrm{sec}$.

In practice, to achieve a reasonable information load, there were two discrimination tasks with different goals presented in a pseudo-random sequence. The cue for starting a trial and for association of the correct goal locus consisted of colourpanels shown above the floor-plan for $2 \mathrm{sec}$ at the start of each trial (Fig. 1). CB requires that during learning a stimulus is added (e.g. $B$ to A). Thus on such a blocking session two colour-panels $(=A)$ were presented up to a learning criterion of $5 / 8$ correct responses, when a third colour-panel (B) was added up to full acquisition (7/8 correct). But blocking performance is judged by comparison with response latencies acquired when all 3 panels were present from the start. Thus there was a second reference session with 3 panels present from the onset of learning: this was presented on the next day counterbalancing our initial study (Oades et al., 1996b). The order of presentation did not contribute to the $\mathrm{CB}$ effect.

At the end of each session single panels that had appeared on the left or right during learning were presented in sequence for 12 test trials. CB measures were derived as follows. The latency of response to the added panel was subtracted from that for the other panel present from the start of learning. This gives the within-session blocking score. Subtraction of the similar value obtained on the reference session (right panel minus left panel latency) from those on the blocking 
session gave the actual CB scores used. Thus the "first trial" measure represents a double subtraction procedure for one discrimination: the first "trial-pair" measure is the mean of the double-subtraction procedures for each of the two discriminations.

Thirty nine patients were unable to meet the learning criteria for the task, even after relaxing the criteria to $7 / 9$ correct within 60 trials and the time for cursor movement from 8 to 10 seconds. (This compares with 3 from 32 patients in the previous study.) The reasons for this lie with the modifications of the task presentation from the original version (Oades et al., 1996b) and are discussed elsewhere (Bender, Müller, Oades, \& Sartory, 2000). An example is the inclusion of extensions on the maze walls to inhibit subjects rushing the cursor through the floor-plan and finding the goal without exhibiting any learning. However, as these patients were able to perform the neuropsychological tests they provide a useful group for the study of the neuropsychological associations of learning the visuo-spatial task in which $\mathrm{CB}$ was tested.

\section{Conditioned Blocking Task (CB)}

Reference Session (start left or mirror-image start right): learning phase ( 3 colours) then
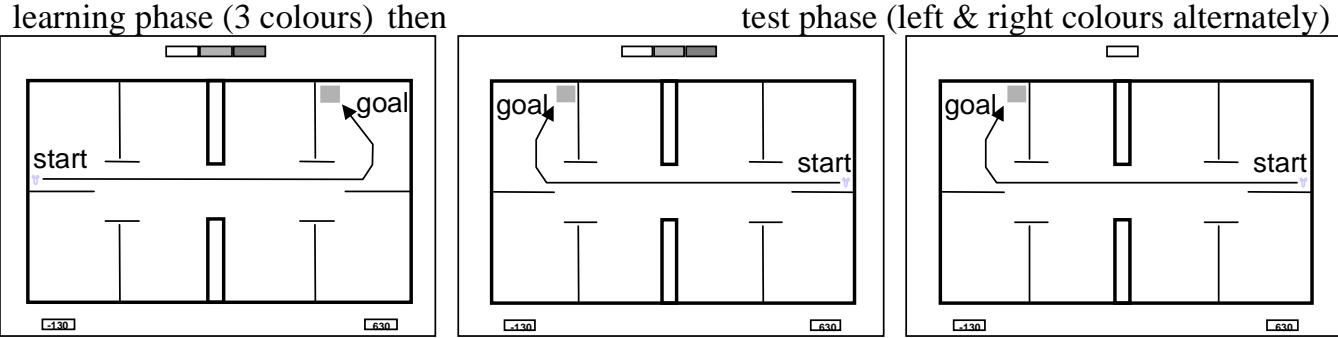

"Blocking" Session (start left or mirror-image start right): learning phase 1 ( 2 colours) then phase 2 ( 3 colours) then
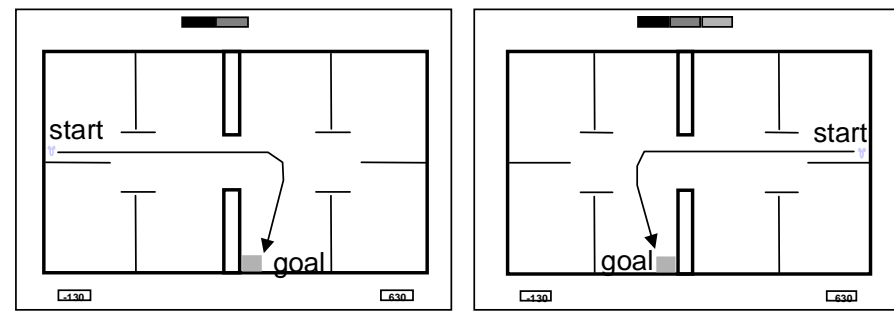

test phase (left \& right colours)

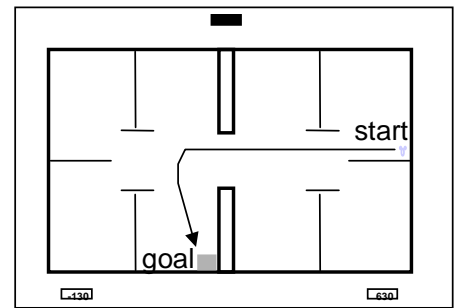

\section{Figure 1}

Schematic drawing of the floor-plan of the CB-task with arrows showing the direct route for the mouse to the goal. On the reference task (top) three colour panels are present through learning and the mouse may start on the left or right (top left and middle floor-plans). The tests phase with a single panel is shown on the right. On the blocking task (bottom) two panels are present in the first learning phase (left floor-plan), then three (middle floor-plan), and then after acquisition the test phase is shown on the right with a single panel. Panels showing the plus and minus points awarded for successful or slow learning, respectively, are shown at the bottom of the floor-plan. 


\section{The Neuropsychological Tasks}

As described in the introduction the neuropsychological test battery consisted of word production on the FAS verbal fluency test and the derived latencies for trail-making (TMT form B-A) and Stroop interference: standard procedures were used for attributing point scores to performance of the blockdesign, picture-completion, Mooney faces closure, visual-reproduction and logical memories (both with immediate and delayed presentations). In addition the advanced progressive matrices (APM: Raven, 1976) were presented in the short 12-item form as an indicator for intelligence. After study of a geometric figure with one part missing, the subject is presented with a choice of 8 patterns for appropriate completion of the figure: whereby 8 correct answers represents average performance $180 \%$ of subjects succeed): scores $<6$ are below and scores of 12 are above average. Handedness was scored according to the Edinburgh inventory (Oldfield, 1971) recording the preference on a scale $0,1,2$ that yields a maximum score of +24 for right- and -24 for left handedness.

\section{Data Treatment}

Subjects were divided by diagnosis (e.g. schizophrenia/ healthy: paranoid/nonparanoid) and group analyses performed. Student t-tests for carried out for demographic and clinical features, and multivariate analyses of variance for experimental variables. As described in the results section the initial MANOVA analyses of group $\mathrm{x}$ performance were modified with covariates for age, IQ and neuroleptic medication as these variables differed between groups and can influence performance (Bender et al., 2000; Oades et al., 2000b). For correlational analyses simple associations were sought with Kendall's tau, as it provides better protection against false positives, more power at sample sizes $>15$ and is less sensitive to violations of statistical assumptions than Pearson's or Spearman's coefficients (Arndt, Andreasen \& Turvey, 1999). Simple correlational analyses were backed up with multiple linear regressions extended in a stepwise fashion. These were performed with respect to the main aims of the study, namely to relate neuropsychological test performance to learning performance, initial and later $C B$ scores: but as the expression of symptoms was related to $C B$, the association between these symptoms and neuropsychological performance was also explored. To maintain the conventional minimum subject/variable relationship of $10: 1$, the reduction of the number of variables for subgroup analyses was based on the structure for the whole group.

The importance of certain symptoms for neuropsychological and CB performance was emphasised through a factor analysis of the symptom ratings with Eigen values larger than one (recommended for 20-50 variables, Hair, Anderson, Tatham, \& Black, 1992). The resultant factor structure used the oblique rotation method that is consistent with the typical clinical situation where patients frequently express symptoms from various dimensions concurrently (Peralta \& Cuesta, 1999). No second order analysis was performed as the result was a clear 5-factor solution. The only minor modification was that only the first 4 factors were considered to be meaningful due to the single item (abstract thought difficulty) in the fifth factor being significantly correlated with the first factor (thought disorder: 0.443). This confirmed the make-up previously reported after varimax rotation (i.e. thought-disturbance, negative symptoms, ideas-of-reference and positive symptoms; Bender et al., 2000).

\section{Figure 2}

The development of blocking (CB) from the first (1-2) to the third trial pair (5-6: sec sem): from left to right, a) in controls (CON) CB decreases to zero with test trials ( $v s$. patients, $\left.\left.\mathrm{SCH},{ }^{*} \mathrm{p}<0.002\right), \mathrm{b}\right) \mathrm{CB}$ is absent initially in nonparanoid patients $(* * p=0.02)$ but is present and persists in those with a paranoid diagnosis, c) a trend for less CB in patients with high vs. low levels of ideas-of-reference (\# $\mathrm{p}=0.07$ ), but $\mathrm{d}$ ) a tendency for more CB in those with high vs. low levels of thought disorder (\# $\mathrm{p}=$ $0.08)$. 


\section{Blocking $(\mathrm{CB})$ in Controls $(\mathrm{CON})$ and Patients $(\mathrm{SCH})$ and Patient Subgroups on first (1-2) vs. last test trial pairs (5-6) \\ paranoid \& nonparanoid diagnoses, low and high scores for the symptom clusters of ideas-of-reference (IoR) and thought disorder}

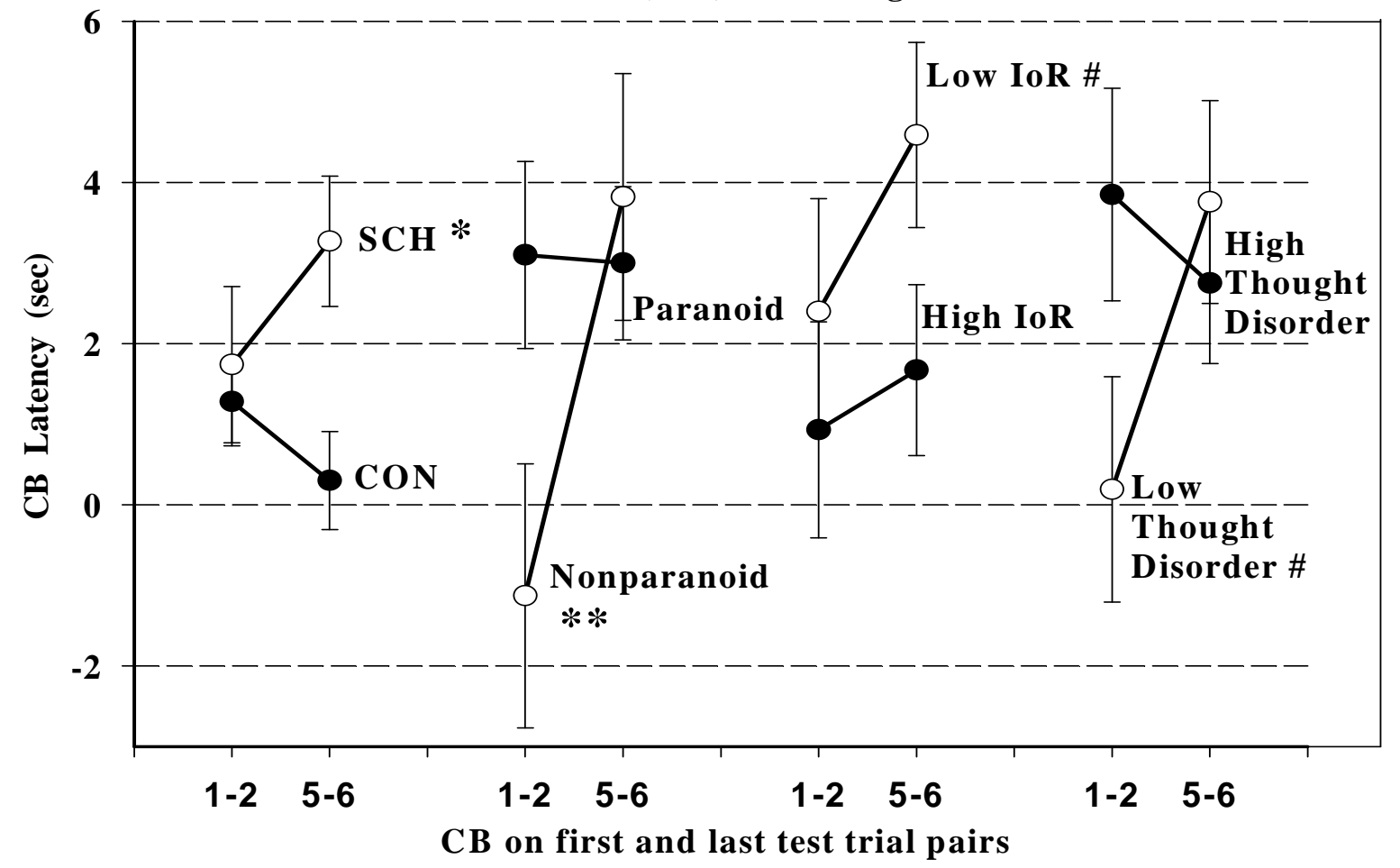

\section{RESULTS}

Patient-control group-comparisons: $\mathrm{CB}$ and neuropsychology

The earlier report of impaired CB in patients with a diagnosis of non-paranoid schizophrenia was replicated (Oades et al., 1996b; 2000: Fig. 2). Reduced CB was associated with some negative symptoms (e.g. poor-rapport and attention). In contrast paranoid patients showed high CB scores and these persisted on test trials (where normally levels decline across test trials) in association with high levels of thought disorder and few ideas-of-reference (Fig. 2).

A comparison of the control and patient groups showed a poorer performance on all neuropsychological tests by the schizophrenia group at the $0.01 \%$ significance level, with the exception of those derived from the Mooney faces closure (see Fig. 3). The present analysis showed no significant differences on any neuropsychological measures when comparing patients with a paranoid with those with a non-paranoid diagnosis.
CB-Task learning abilities: contribution of neuropsychological indices:

Differences were evident between the patients that did and did not learn the task on all neuropsychological tests with the exceptions of the Mooney faces closure and Stroop-interference measures. As the two patient groups differed in age and APM-IQ (Table 1) and individual doses of neuroleptic medication could confound investigations of group differences in psychological measures, all three items were entered as covariates in a MANCOVA on the 10 main neuropsychological measures $[\underline{F}(10,93)=4.13 ; \underline{P}=.0001]$. Posthoc analysis showed that the patients who learned the CB-task adequately were better than those who did not attain the learning criterion on the TMT, picture completion, block-design and visual reproduction tasks (immediate and delayed forms). Significant differences on verbal fluency and prose reproduction (immediate and delayed forms) were reduced to trends with the use of covariates (6-8\% significance, Fig. 3). 
Thus the functions pertinent to learning the CB-task involved cognitive flexibility (TMT, block-design) and visuo-spatial, perceptuomotor abilities (picture completion and pattern reproduction). The neural systems whose functions are implicated are those associated with the frontal lobes and the right hemisphere.

Entering performance on these neuropsychological tests into a step-wise regression on the number trials required to reach the learning criterion on the CB-task confirms the role of visuo-spatial abilities of the right hemisphere. Thus, for the reference learning session the last task left in the equation was block-design $[\underline{F}(1,60)=5.1, \underline{P}=$ $\left..027, R^{2} 0.078 ; \beta-0.28\right)$. For the two learning phases on the blocking session, the last task left in the equation was, respectively, visualreproduction immediate and delayed form [F $(1,60)=1.2 / 9.2, \underline{P}=0.0002 / 0.003, R^{2} 0.21 /$ $0.13, \beta-0.46 /-0.36]$.

\section{Figure 3}

Representative measures of neuropsychological task performance are shown for controls (CON) and patients that did (SCH/learn) or did not successfully acquire the associative learning task ( $\mathrm{SCH} /$ notlearn). Increasing latencies (sec, $S D$ ) show impairments on the two left-hand tasks, while on the right fewer points illustrate impairments of performance $(S D)$. All comparisons except Mooney-Faces were significant between patients and controls $(<1 \%)$. Comparisons between patient groups are shown as follows, \# $0.09>p>0.06, * p=0.04, * * p=0.01$, *** $p<0.001$.

Verbal Fluency, Mooney-Faces, Picture Completion, Block-Design, with Visual Reproduction (+ Delay) and Prose-Reproduction (+ Delay)

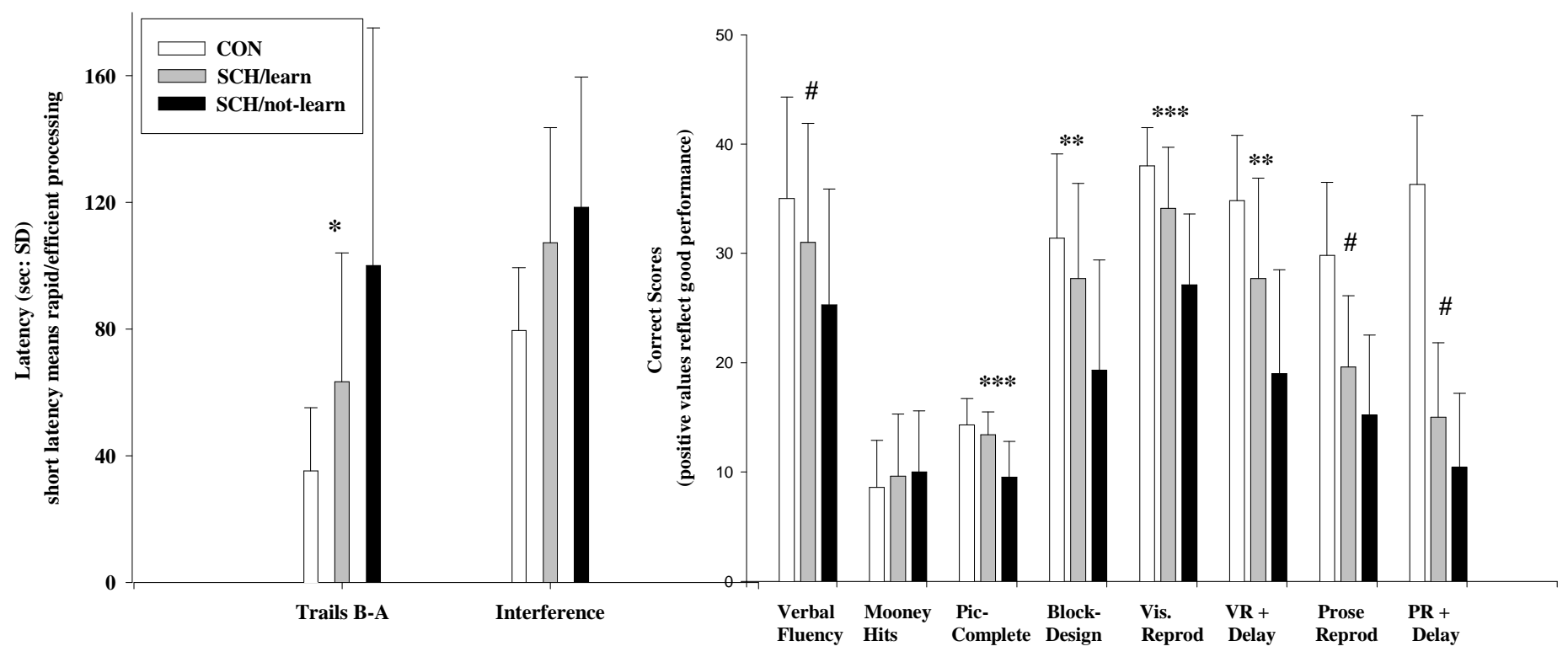


CB:

Contribution of neuropsychological indices:

A multiple linear regression for the 10 main neuropsychological measures with $C B$ in the 124 subjects learning the task explained significantly $15.2 \%$ of the variance $[F(10,113)$ $=2.02, \underline{P}=0.03]$. Beta-weights were significant for Stroop interference and Mooney-faces hits $(\beta+0.23, \underline{P}=0.02 \& \beta+0.19, \underline{P}=0.04$, respectively). Trends were also noted for verbal fluency, immediate visual reproduction and picture completion (see Figure 4, partial correlations). The same model was significant for patients who learned the CB-task $[\mathrm{F}(10,51)$ $=1.98, \underline{P}=0.05]$, but, given the desirable 1:10 ratio between subjects and measures in such a regression, the variables were reduced to 6 by a stepwise procedure for these 62 patients [ $\mathrm{F}$ $(6,55)=3.1, \underline{P}=0.01]$. Significant positive contributions to $C B$ were made by performance on verbal fluency, Stroop interference, Mooney faces hits, visual reproduction ( $B$ 0.12-0.16, $\underline{\mathrm{P}}=0.02-0.05$ ) with the tendency of a negative contribution from delayed visual reproduction ( $\beta-0.3, \underline{P}=0.057$; $R^{2}=0.25$ : see Fig. 4). Regression analyses were not significant for $C B$ scores late in the test phase, when patients with paranoid schizophrenia showed higher than normal levels of $\mathrm{CB}$. There was a non-significant trend for Stroop-interference to continue to show an association (partial correlation, +0.28 ).
Thus, in addition to the visuospatial functions of the right-hemisphere advantageous to acquiring the task, parietal, cingulate and perhaps left frontal functions relating to flexibility, effort and selective processing seem to relate to $C B$ performance.

\section{Symptoms dimensions and task performance:} Was neuropsychological performance associated with the main symptom dimensions? The factor-analysis confirmed the four main symptom dimensions previously reported for these patients (i.e. thoughtdisturbance, negative symptoms, ideas-ofreference and positive symptoms; Bender et al., 2000). But, the regressions for these symptom dimensions on $C B$ scores did not prove significant. Nonetheless it is of interest to note that these symptom dimensions correlated with neuropsychological performance as follows: a) thought disorder with poor visual reproduction and block design (right hemisphere function: $\tau=-0.2$, $\underline{P}$ $<.05)$; b) negative symptoms with indicators of left frontal and temporal lobe function (TMT [ $\tau=+0.2]$, prose recall $[\tau=-0.17]$ and verbal fluency $[\tau=-0.26 ; .025>\underline{\mathrm{P}}>.0005)$; c) positive symptoms with poor prose-recall (left temporal lobe function: $\tau=-0.17, \underline{\mathrm{P}}<0.05)$, and $d)$ ideas-of-reference tended to be negatively associated with verbal fluency (left frontal lobe function; $\tau=-0.25$ ). 
Partial Correlation Coefficients for Neuropsychological Test Performance on CB:

(a) all 124 subjects, \& (b) 61 patients on 10 main measures,

(c) replicated for patients with 6 measures in regression.

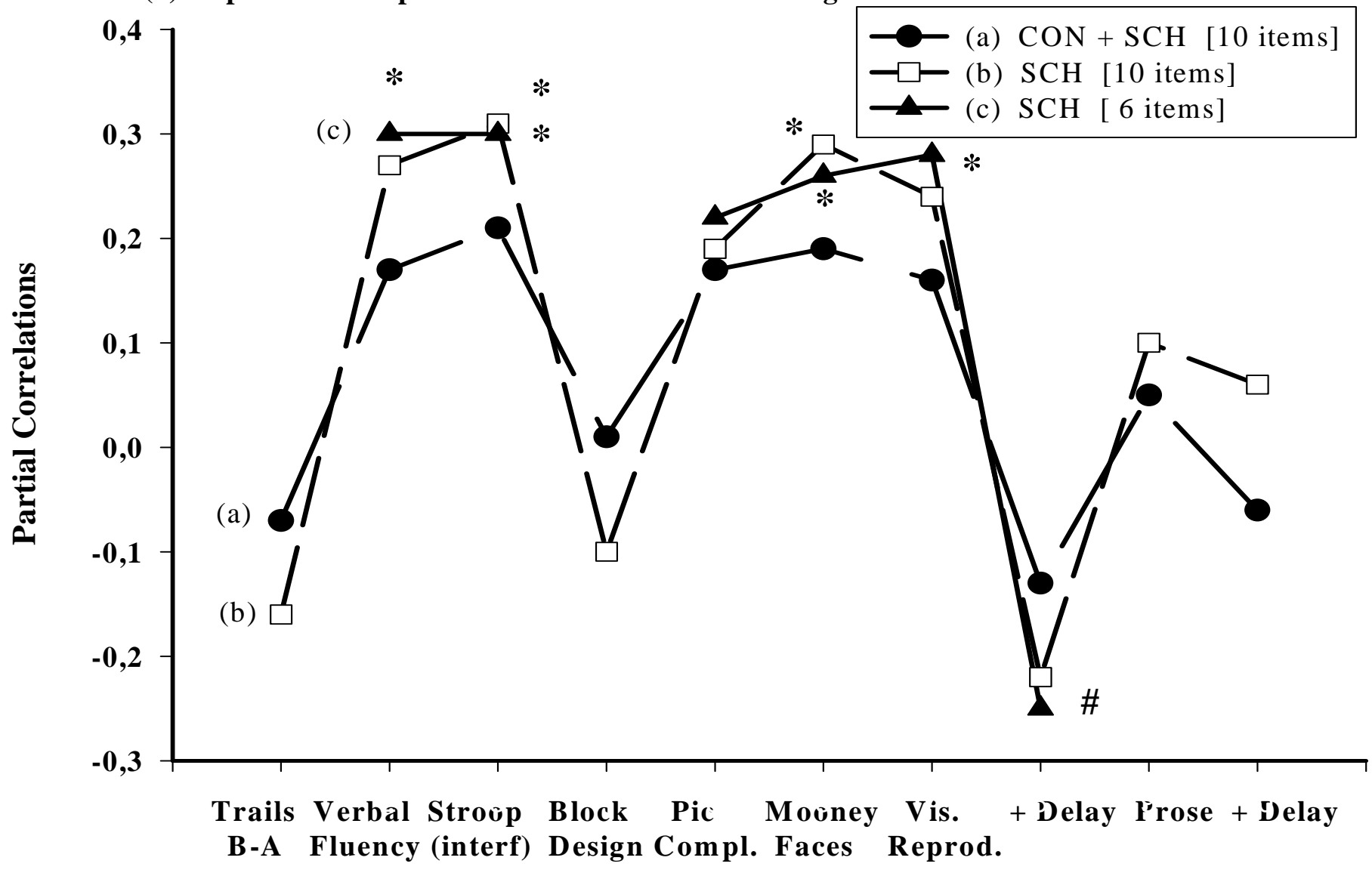

Figure 4

Partial correlation coefficients are charted for performance on 10 neuropsychological tasks for initial conditioned blocking (CB). The circles show the strength of the relationship for all subjects $(n=124)$, the squares show the relationship for the patients alone $(n=61)$, and the triangles illustrate the relationship for 6 items determined by stepwise backward regression.

$\# \mathrm{p}=0.057, * 0.05<\mathrm{p}<0.02$

\section{DISCUSSION}

Associations for the performance of $C B$ with 10 neuropsychological tests were sought for the indication of the nature of function and the type of cortical region likely to be involved in learning and in the unusual levels of $C B$ shown by groups of patients with schizophrenia. Good block-design scores related to faster associative learning in all subjects. In patients there was an additional association with immediate and delayed visual reproduction. This association of visual perceptual and problem solving abilities with the learning of a visuo-spatial task makes good sense, and implicates frontal, parietal and temporal lobe function especially in the right hemisphere. The comparison between patient groups that learned and did not learn the task adequately confirmed the importance of these abilities, but additionally showed up differences of trail-making, picture completion and a trend difference in verbal fluency. This result puts the emphasis on flexible, conceptual problem-solving abilities involved in the task, namely the need to switch between the two discriminations, try alternative goal localities, and shift between the perceptual levels of analysis and sets involved. This emphasis points to an important contribution from frontal lobe activity in the left hemisphere.

The second set of findings showed that $\mathrm{CB}$ measures of selective attention depended, in addition to the continued role of visual 
reproduction and verbal fluency (right temporal and left frontal lobe function), on Stroop interference effects and success on the Mooney faces closure test. These latter two tasks emphasise a CB association, respectively, with the effort incurred in mastering the conflict between automatic and controlled attentional strategies (Stroop-test, Hartje \& Poeck, 1997), and the need to use holistic perceptual abilities (Mooney-faces, Lezak, 1995). Structurally speaking the effortful evaluation of stimuli implies an active contribution from the anterior cingulate region of the frontal lobe (Stroop, Kolb \& Whishaw, 1996, Cabeza \& Nyberg, 1997). In addition the right parietal cortex implicated in the Mooney-faces test (Wasserstein, Zappulla, \& Rosen, 1987) has been repeatedly associated with abilities to manipulate visuospatial material (Harris, Egan, Sonkkila, Tochon-Danguy, Paxinos et al., 2000 and references therein) and the allocation of visuospatial attention (Mesulam, 1999).

Age, illness-onset age and IQ can influence the performance of tasks described here in a small way (Bender et al., 2000). Young age tended to have a positive but early-onset a negative influence on task learning. Patients who did not acquire the CB-task had a lower performance-IQ. However, age-related influences were controlled for by covariance, and $C B$ measures were studied for patient and control groups who had learned the task and were closely matched on a case by case basis.

Were the results influenced by the medication status and the differences in the type of antipsychotic drug administered? There are many reports of neuropsychological impairment in patients with schizophrenia, especially on memory-related measures, that also claim that medication had little influence (e.g. Aleman, Hijman, de Haan, \& Kahn, 1999). However, these have mostly relied on relating the performance data to treatment in terms of chlorpromazine equivalents (CPZ). We have recently confirmed the claim in terms of $C P Z$, but shown the confidence to be misplaced when antipsychotic medication is calculated in terms of serum levels of dopamine-D2 binding activity or estimated levels of central D2 occupancy (Oades, Rao, Bender, Sartory, \&
Müller et al., 2000b). We have shown that increased dopamine activity is positively associated with the expression of $\mathrm{CB}$ (Oades et al., 1996b, 2000b). This is the opposite result to those obtained for LI. Further, we showed that measures of dopamine D2 binding activity related positively to block design and prose recall, but negatively with trail making, verbal fluency and picture-completion. Indeed, there are several recent reports that medication may improve performance on tests of attention, interference, verbal fluency, visual recall and Mooney-faces (Buchanan, Holstein \& Breier, 1994; Verdoux, Magnin \& Bourgeois 1995). Some authors differ with respect to specific tests and note some negative influences of treatment in general or of specific neuroleptics for card-sorting and memorial functions (Michel, Danion, Grange, \& Sandner 1998; Earnst, Taylor, Smet, Goldman et al., 1999). Thus, although medication (CPZ) was used as a covariate in the current comparisons of patient groups, it may not have had as large a controlling effect as intended. Nonetheless it should be emphasised that 'qualitatively' similar neuropsychological profiles are reported for medicated and unmedicated patients on such tests (Saykin, Shtasel, Gur, Kester, Mozley et al., 1994).

With the plausible contributions of righthemisphere abilities to learning a visuo-spatial discrimination, and left frontal function to problem solving (described above), the context of the association of Stroopinterference and Mooney-Faces test to $C B$ should be considered. Interpretations of the Stroop interference effect include 'conflicts in perceptual coding', 'response competition', 'inherent property of parallel processing' and 'conflicts for more automatic reading vs. less automatic naming' (Peterson, Skudlarksi, Gatenby, Zhang et al., 1999). The latter explanation is preferred here because of the undoubted association of the cingulate region in attentional processing that requires effort an uncontroversial characteristic of interference test performance (Mesulam, 1999). There is considerable consistency from early PET to recent $f M R I$ studies of this effect in identifying activation around the anterior cingulate region, and recently de-activation in 
mid-temporal lobe regions (Pardo, Pardo, Janer, \& Raichle, 1990; Peterson et al., 1999). This too is remarkably consistent with reports of exaggerated Stroop interference in the disorganised (thought-disordered) subgroup of schizophrenia that also showed anomalously increased cerebral blood flow in the anterior cingulate (Liddle \& Morris, 1991). Further, in the current data thought disordered symptoms correlated well with the expression of initial $C B$ and its persistence across test presentations.

A recent examination of problem-solving behavioural strategies in schizophrenia found that experimental indicators of thought disorder predicted the dysregulation of response sequences (Paulus, Perry \& Braff, 1999). The authors' analysis demonstrated that an accurate perception and processing of the subjects' experience of the previous situation was disturbed, and led to this dysregulation. It is precisely this ability to refer to stored regularities that Hemsley (1994) saw as disturbed in many patients of schizophrenia, especially those with positive thought disorder, and described as the difficulty to disengage from automatic processing when controlled information processing was required. It is also this ability that is normally called for when an added stimulus has been ignored during associative learning (CB), but is then presented alone for response on a series of trials during the course of testing.

The sensitivity of the Mooney-faces-closure test to visuo-spatial holistic processing (Wasserstein et al. 1987) partly explains the association with $C B$ measures on the visuospatial mouse-in-house task with its navigation requirements. More precisely the association concerns the function of the parietal cortex (Milner, 1980). The parietal component of networks mediating attentional processing lies with the 'perceptual representation of behaviourally relevant extra-personal events as targets for attentional behaviours' (Mesulam, 1999). Again recent $\mathrm{fMRI}$ studies have illuminated some of the details of function relevant to $C B$ in associative learning. Thus, featureconjunction or binding (physical associative properties of attention, Treisman, 1988) lead to specific activation of superior parietal cortex (Corbetta, Shulman, Miezin, \& Petersen, 1995) and similar features in the temporal domain have resulted in activation in the intra-parietal sulcus (Coull, Frith, Frackowiak, \& Grasby, 1997). We are only aware of one group reporting on the performance of patients with schizophrenia on the Mooney-faces test. They found that patients with the deficit syndrome were more impaired than those without it (Buchanan et al., 1994). However, although certain negative symptoms in the current study were associated with reduced $C B$, there is little resemblance with the group studied by Buchanan and colleagues, firstly as there were no group differences for our patients on the Mooney-faces test, and secondly their subjects were, unlike our own, unimpaired on the block-design task.

In conclusion, we describe a potential contribution of function on 4 of 10 neuropsychological measures, to the expression of $C B$ measures of selective attention on a visuospatial associative learning task. This was based on a comparison of the performance of patients with schizophrenia with healthy controls. These functions are thought to reflect activity in the right parietal, cingulate and left frontal cortices. The frontal and cingulate contributions to associative learning measures are consistent with other studies of the effects of focal frontal damage on associative learning (Levine, Stuss, \& Milberg, 1997). However, the novel aspect is the association with activity in cingulate and parietal regions that contribute to known attentional networks (Mesulam, 1999). More usually schizophrenic deficits are emphasised for trail-making and word fluency (here, relevant to task learning) and for verbal/nonverbal memory, that did not play a significant role in the current analysis of attentionrelated mechanisms (e.g. Heinrichs \& Zakanis, 1998). While the associations might have been stronger, similar results are reported in other regression (vs. categorical) analyses of neuropsychological data from schizophrenics (Verdoux, et al., 1999). The variance that remains unexplained in the attribution of structure and function contributing to $C B$ from 
neuropsychological performance may in part reflect the imprecision inherent in neuropsychological tests, and could be reduced by extending the test-battery, or, with regard to brain regions, applying modern functional imaging techniques.

\section{References:}

Aleman, A., Hijman, R., de Haan, E.H.F., \& Kahn, R.S. (1999). Memory impairment in schizophrenia: a meta-analysis. American Journal of Psychiatry, 156, 1358-1366.

Allen, D.N., Goldstein, G., \& Aldarondo, F. (1999). Neurocognitive dysfunction in patients with schizophrenia and alcoholism. Neuropsychology, 13, 62-68.

American Psychiatric Association, (1994). Diagnostic and Statistical Manual of Mental Disorders (DSM-IV), $4^{\text {th }}$ ed., Washington, D.C.: American Psychiatric Association.

Andreasen, N.C., \& Olsen, S. (1982). Negative versus positive schizophrenia: definition and validation. Archives of General Psychiatry, 39, 789-794.

Arndt, S., Turvey, T., \& Andreasen, N.C. (1999). Correlating and predicting psychiatric symptoms ratings: Spearman's $r$ versus Kendall's tau correlation. Journal of Psychiatric Research, 33, 97-104.

Barnes, T.R.E. (1989). A rating scale for the drug-induced akathisia. British Journal of Psychiatry, 154, 672-676.

Bender, S., Müller, B.W., Oades, R.D., \& Sartory, G. (2000). Conditioned blocking and schizophrenia: a replication and study of the role of symptoms, age, illness onsetage and illness duration. Schizophrenia Research, in press.

Benton, A.L., \& Hamsher, K. (1989). Multilingual Aphasia Examination. Iowa City, IA: AJA Associates.

Brauns, H., Haun, D. and Steinmann, S., 1997. The construction of an internationally comparable classification by class. Erwerbsstatistische Besonderheiten am Beispiel von Labour Force Surveys der Bundesrepublik Deutschland, Frankreichs, Großbritanniens und Ungarns., Mannheim Centre for European Social Research (MZES), Mannheim: Arbeitspapiere Arbeitsbereich 1/22.

Botvinick, M., Nystrom, L.E., Fissell, K., Carter, C.S., \& Cohen, J.D. (1999). Conflict monitoring versus selection-for-action in anterior cingulate cortex. Nature, 402, 179181.

Buchanan, R.W., Holstein, C., \& Breier, A. (1994). The comparative efficacy and longterm effect of clozapine on neuropsychological test performance. Biological Psychiatry, 36, 717-726.

Cabeza, R., \& Nyberg, L. (1997). Imaging cognition: an empirical review of PET studies with normal subjects. Journal of Cognitive Neuroscience, 9, 1-26.

Callaway, E., \& Naghdi, S. (1981). An information processing model of schizophrenia. Archives of General Psychiatry, 38, 339-347.

Caza, P.A. (1984). Noradrenergic influences on blocking: interactions with development. Pharmacology Biochemistry and Behavior, 21, 9-17.

Chase, T.N., Fedio, P, \& Foster, N.L. (1984). Wechsler Adult Intelligence Scale performance. Cortical localization by fluorodeoxyglucose F18 positron emission tomography. Archives of Neurology, 41, 1244-1247.

Corbetta, M., Shulman, G.L., Miezin, F.M., \& Petersen, S.E. (1995). Superior parietal cortex activation during spatial attention shifts and visual feature conjunction. Science, $270,802-805$

Coull, J.T., Frith, C.D., Frackowiak, R.S.J., \& Grasby, P.M. (1997). A fronto-parietal network for rapid visual information processing: a PET study of sustained attention and working memory. Neuropsychologia, 34, 1085-1095.

Crider, A., Blockel, L., \& Solomon, P.R. (1986). A selective attention deficit in the rat following induced dopamine receptor supersensitivity. Behavioral Neuroscience, 100, 315-319.

Delaney, R.C., Rosen, A.J., Mattson, R.H., \& Noelly, R.A. (1980). Memory function in focal epilepsy: a comparison of nonsurgical, unilateral temporal lobe and frontal lobe samples. Cortex, 16, 103-117.

Dove, A., Pollmann, S., Schubert, T., Wiggins, C.J., \& von Cramon, D.Y. (2000). Prefrontal cortex activation in task switching: an event-related fMRI study. Cognitive Brain Research, 9, 103-109. 
Earnst, K.S., Taylor, S.F., Smet, I.C., Goldman, R.S., Tandon, R., Berent, S. (1999). The effects of typical antipsychotics, clozapine and risperidone on neuropsychological test performance in schizophrenia. Schizophrenia Research, 40, 255-256

Fink, G.R., Halligan, P.W., Marshall, J.C., Frith, C.D., Frackowiak, R.S.J., \& Dolan, R.J. (1996). Where in the brain does visual attention select the forest and the trees? Nature, $382,626-628$.

Gray, J.A. (1998). Integrating schizophrenia. Schizophrenia Bulletin, 24, 249-266.

Gray, N.S., Pickering, A.D., Gray, J.A., Jones, S.H., Abrahams, S.H., \& Hemsley, D.R. (1997). Kamin blocking is not disrupted by amphetamine in human subjects. Journal of Psychopharmacology, 11, 301-311.

Hair, J.F., Anderson, R.E., Tatham, R.L., \& Black, W.C. (1992). Multivariate data analysis. (3rd Ed.) New York: Macmillan.

Harris, I.M., Egan, G.F., Sonkkila, C., TochonDanguy, H.J., Paxinos, G., \& Watson, J.D.G. (2000). Selective right parietal lobe activation during mental rotation. Brain, 123, 65-73.

Hartje, W., \& Poeck, K. (1997). Klinische Neuropsychologie. Stuttgart: Georg Thieme Verlag.

Heinrichs, R.W., \& Zaksanis, K.K. (1998). Neurocognitive deficit in schizophrenia: a quantitative review of the evidence. Neuropsychology, 12, 426-445.

Hemsley, D.R. (1994). A cognitive model for schizophrenia and its possible neural basis. Acta Psychiatrica Scandinavica, 90 (supplement 384), 80-86.

Holland, P.C. (1999). Overshadowing and blocking as acquisition deficits: no recovery after extinction of overshadowing or blocking cues. Quarterly Journal of Experimental Psychology, 52B, 307-333.

Hidaka, T., Sadato, N., Yamada, H., \& Yonekura, Y. (2000). Functional asymmetry of human prefrontal cortex in verbal and non-verbal episodic memory as revealed by fMRI. Cognitive Brain Research, 9, 73-83.

Kamin, L.J. (1969). Predictability, surprise. Attention and conditioning. In R. Church \& B. Campbell (Eds.), Punishment and aversive behavior (pp. 279-296). New York, NY: Appleton-Century-Crofts.
Kay, S.R., Opler, L.A., \& Fizbein, A. (1992). The Positive and Negative Syndrome Scale (PANSS). Toronto: Manual. Multi-Health Systems.

Kim, Y-H., Gitelman, D.R.., Nobre, A.C., Parrish, T.B., LeBar, K.S., \& Mesulam, M-M. (1999). The large scale neural network for spatial attention displays multifunctional overlap but differential asymmetry. Neurolmage, 9, 269-277.

Kleinschmidt, A., Büchel, C., Zeki, S., \& Frackowiak, R.S.J. (1998). Human brain activity during spontaneously reversing perception of ambiguous figures. Proceedings of the Royal Society of London B 265, 2427-2433.

Kolb, B., \& Whishaw, I.Q. (1996). Neuropsychologie. Heidelberg: Spektrum Akademischer Verlag.

Kumari, V., Cotter, P.A., Mulligan, O.F., Checkley, S.A., Gray, N.S., Hemsley, D.R., Thornton, J.C., Corr, P.J., Toone, B.K., \& Gray, J.A. (1999). Effects of d-amphetamine and haloperidol on latent inhibition in healthy male volunteers. Journal of Psychopharmacology, 13, 398-405.

Lansdell, H. (1970). Relation of extent of temporal removals to closure and visuomotor factors. Perceptual and Motor Skills, 31, 491-498.

Levine, B., Stuss, D.T., \& Milberg, W.P. (1997). Effects of aging on conditional associative learning: process analyses and comparison with focal frontal lesions. Neuropsychology, 11, 367-381.

Lezak, M.D. (1995). Neuropsychological Assessment. New York: Oxford University Press.

Liddle, P.F. \& Morris, D.L. (1991). Schizophrenic syndromes and frontal lobe performance. British Journal of Psychiatry, 158, 340-345.

Mesulam, M-M. (1999). Spatial attention and neglect: parietal, frontal and cingulate contributions to the mental representation and attentional targeting of salient extrapersonal events. Philosophical Transactions of the Royal Society series B, 354, 13351346.

Michel, L., Danion, J-M., Grange, D., \& Sandner, G. (1998). Cognitive skill learning and schizophrenia: implications for 
cognitive remediation. Neuropsychology, 12, 590-599.

Mooney, C.M., \& Ferguson, G.A. (1951). A new closure test. Canadian Journal of Psychology, 5, 129-133.

Oades, R.D., Röpcke, B., \& Schepker, R. (1996a). A test of conditioned blocking and its development in childhood and adolescence: relation-ship to personality and monoamine metabolism. Developmental Neuropsychology, 12, 207-230.

Oades, R.D., Zimmermann, B., \& Eggers, C. (1996b). Conditioned blocking in patients with paranoid, non-paranoid psychosis or obsessive compulsive disorder: Association with symptoms, personality and monoamine metabolism. Journal of Psychiatric Research, 30, 369-390.

Oades, R.D., Müller, B.W., Bender, S., \& Sartory, G. (2000a). A conditioned blocking (CB) impairment in nonparanoid schizophrenia: onset-age and illness duration. Schizophrenia Research, 41, 292293.

Oades, R.D., Rao, M.L., Bender, S., Sartory, G., \& Müller, B.W. (2000b). Neuropsychological and conditioned blocking performance in patients with schizophrenia: assessment of the contribution of neuroleptic dose, serum levels and dopamine D2-receptor occupancy. Behavioural Pharmacology, 11, 317-330.

Oades, R.D., Rivet, J-M., Taghzouti, K., Kharouby, M., Simon, H. \& Le Moal, M. (1987). Catecholamines and conditioned blocking: effects of ventral tegmental, septal and frontal 6-hydroxydopamine lesions in rats. Brain Research, 406, 136146.

Oldfield, R.C. (1971). The assessment and analysis of handedness: the Edinburgh Inventory. Neuropsychologia, 9, 97-113.

Pardo, J.V., Pardo, P.J., Janer, K.W., \& Raichle, M.E. (1990). The anterior cingulate mediates processing selection in the Stroop attentional conflict paradigm. Proceedings of the National Academy of Sciences (USA), 87, 256-259.

Paulus, M.P., Perry, W., \& Braff, D.L. (1999). The nonlinear, complex sequential organization of behavior in schizophrenic patients: neurocognitive strategies and clinical correlations. Biological Psychiatry, 46, 662-670.

Peralta, V. Cuesta, \& M.J. (1999). Dimensional structure of psychotic symptoms: an itemlevel analysis of SAPS and SANS symptoms in psychotic disorders. Schizophrenia Research, 38, 13-26.

Perret, E. (1974). The left frontal lobe of man and the suppression of habitual responses in verbal categorical behaviour. Neuropsychologia, 12, 323-330.

Peterson, B.S., Skudlarski, P., Gatenby, J.C., Zhang, H., Anderson, A.W., \& Gore, J.C. (1999). An fMRI study of Stroop word-color interference: evidence for cingulate subregions subserving multiple distributed attentional systems. Biological Psychiatry, 45, 1237-1258.

Raven, J.C. (1976). Raven-Matrizen-Test: Advanced Progressive Matrices. German language manual. $\mathrm{H}$. Kratzmeier and $\mathrm{R}$. Horn (Eds.). Stuttgart: Beltz-Verlag.

Reitan, R.M. (1958). The validity of the Trail Making Test as an indicator of organic brain damage. Perceptual and Motor Skills, 8, 271-276.

Rickert, E.J., Bennett, T.L., Lane, P., \& French, J., (1978). Hippocampectomy and the attenuation of blocking. Behavioral Biology, 22, 147-160.

Saykin, A.J., Shtasel, D.L., Gur, R E., Kester, D.B., Mozley, L.H., Stafiniak, P. \& Gur, R.C. (1994). Neuropsychological deficits in neuroleptic naive patients with first episode schizophrenia. Archives of General Psychiatry, 51, 124-131.

Simpson, G.M., \& Angus, J.W.S. (1970). A rating scale for extrapyramidal side-effects. Acta Psychiatrica. Scandinavica, supplement 212, 11-19.

Spence, S.A., Liddle, P.F., Stefan, M.D., Hellewell, J.S.E., Sharma, T., Friston, K.J., Hirsch, S.R., Frith, C.D., Murray, R.M., Deakin, J.F.W., \& Grasby, P.M. (2000). Functional anatomy of verbal fluency in people with schizophrenia and those at genetic risk: focal dysfunction and distributed disconnectivity reappraised. British Journal of Psychiatry, 176, 52-60.

Straube, E.R., \& Oades, R.D. (1992). Schizophrenia: Empirical Research and Findings. San Diego: Academic Press. 
Taylor, S.F., Kornblum, S., Lauber, E.J., Minoshima, S., Koeppe, R.A. (1997). Isolation of specific interference processing in the Stroop task. PET activation studies. Neurolmage, 6, 81-92.

Treisman, A.M. (1988). A feature-integrative theory of attention. Quarterly Journal of experimental Psychology, 40, 201-237.

Verdoux, H., Magnin, E., \& Bourgeois, M. (1995). Neuroleptic effects on neuropsychological test performance in schizophrenia. Schizophrenia Research, 14, 133-139.

Verdoux, H., Liraud, F., Gonzales, B., Assens, F., Abalan, F., Beaussier, J-P., Gaussares, C., Etchegarry, B., Bourgeois, M.L., \& van Os, J. (1999). The association of neuropsychological deficits to clinical symptoms in first-admission subjects with psychotic disorders. Schizophrenia Research, 37, 197-203.

Warrington, E. K., James, M., \& Maciejewski, C. (1986). The WAIS as a lateralizing and localising diagnostic instrument. Neuropsychologia, 24, 223-239.

Wasserstein, J., Zappulla, R., \& Rosen, J. (1987). In search of closure: Subjective contour illusions, gestalt completion tests and implications. Brain and Cognition, 6, 114.

Wechsler, D. (1981). Wechsler Adult Intelligence Scale-revised (WAIS-R). New York, NY: The Psychological Corporation.

Wechsler, D. (1987). The Wechsler Memory Scale-Revised. San Antonio, TX: The Psychological Corporation, Harcourt Brace Jovanovich. 
